\title{
Coping and depression in diabetic foot ulcer healing: causal influence, mechanistic evidence or none of the above?
}

\author{
J. S. Gonzalez • M. J. Hardman • A. J. M. Boulton • \\ L. Vileikyte
}

Received: 6 July 2010 /Accepted: 16 August 2010 /Published online: 13 October 2010

(C) Springer-Verlag 2010

Keywords Coping $\cdot$ Depression $\cdot$ Diabetes $\cdot$ Foot ulcers

To the Editor: We read with interest the article by Vedhara and colleagues on coping, depression and healing of diabetic foot ulcers [1], as we believe the investigation of psychosocial aspects of foot ulceration is important and understudied. However, we were disappointed that claims of 'influence' and 'mechanistic evidence' in the title and conclusions of the paper do not appear to be supported by the data presented. Although problems regarding the reliability of depression and avoidance coping measures, and small sample sizes for secondary analyses are noted, other important methodological problems are not.

First, the presented data provide no mechanistic evidence, but merely demonstrate that indicators of cortisol and matrix metalloproteinase (MMP) are associated with ulcer healing. It is not reported whether these potential mediators were associated with the predictors in question (i.e. depression and confrontational coping), or

J. S. Gonzalez $(\bowtie)$

Ferkauf Graduate School of Psychology, Yeshiva University,

Rousso Building, 1300 Morris Park Avenue,

Bronx, NY 10461, USA

e-mail: jeffrey.gonzalez@einstein.yu.edu

\section{J. S. Gonzalez}

Albert Einstein College of Medicine,

Bronx, NY, USA

M. J. Hardman · A. J. M. Boulton · L. Vileikyte

University of Manchester,

Manchester, UK

A. J. M. Boulton - L. Vileikyte

University of Miami,

Miami, FL, USA whether controlling for the purported mechanisms attenuated the relationship between the predictors and outcome [2]. As the authors had these data at their disposal, they could and ought to have evaluated the relationships between depression and coping, and cortisol and MMP, which they allude to in their discussion. Restricting their analyses to those with complete data on these variables would also have addressed the possibility that the reported differences in the associations between confrontational coping and depression, and ulcer healing and change in ulcer area may have been due to chance variations in the relationships between these variables across quite different samples (e.g. $n=93$ versus 21 ). It is also unclear why the authors stress a relationship between the pro-form of MMP2 and ulcer healing (the direction of which is misstated in the abstract). Wound fluid levels of the active form of this extracellular protease would be of functional significance, yet crucially no difference in active MMP2 levels was observed. Detailed histological analysis (e.g. in situ zymography) would have clarified this discrepancy. Importantly, depression was not associated with ulcer healing, but with change in ulcer area; the question of whether cortisol or MMP indices were related to change in ulcer area was not addressed. Thus, no data presented link these potential 'mechanisms' to depression or change in ulcer area.

Second, the analytical approach could have affected important analyses. The authors note that there were no a priori hypotheses regarding which of three coping styles would be associated with their primary or secondary outcomes. There were also no predictions about which of five indicators of cortisol or four indices of MMP would be related to the two ulcer healing measures. Lack of hypotheses or control for familywise error may substantially increase the chance of spurious findings [3]. Over-fitting, or having too many predictor variables for a given sample 
size, is also likely to be problematic in the primary analysis of ulcer healing [4]. This analysis by Vedhara and colleagues [1] includes 37 non-healed ulcer events predicted by nine independent variables, for an event/predictor ratio of $4: 1$. This is well below the recommended ratio of 10:1 [4] and problematic even if the recommended ratio were relaxed [5]. It would be reassuring if the relationship between confrontation coping and ulcer healing were significant at the bivariate level or in a less over-fitted multivariate model.

Finally, we have concerns regarding definitions of neuropathy and ischaemia. The authors cite a previous paper [6] in justifying their classification of neuropathy using monofilament insensitivity. This is inaccurate, as no mention of monofilaments is made in this paper [6]; rather, the modified neuropathy disability score was used. Monofilaments are used to identify risk of ulceration rather than in neuropathy assessment. Moreover, the authors fail to state which sites were tested and how abnormalities were defined. With respect to ischaemia, it is stated that patients were ineligible if no palpable pulses were present, yet descriptive data for ischaemia severity are reported and ischaemia is included as a covariate in multivariate analyses. Clarification of these discrepancies would be helpful.

Research on the relationship between psychosocial variables and foot ulcer outcomes with a focus on identifying the underlying mechanisms is important. However, the significance of physiological mechanisms may be difficult to determine without accounting for the role of behaviour. This is especially relevant to the study of diabetic foot ulcers, where continued pressure on plantar foot lesions as a result of non-adherence to offloading is a major cause of ulcer chronicity. For example, histological examination reveals improved healing (angio- genesis and granulation tissue formation) in patients receiving off-loading compared with increased local inflammation in specimens from patients without offloading [7]. In sum, although Vedhara and colleagues claim to provide evidence for physiological links between psychological factors and ulcer outcomes, we feel that their conclusions are premature and could lead others down unrewarding paths of investigation.

Duality of interest The authors declare that there is no duality of interest associated with this manuscript.

\section{References}

1. Vedhara K, Miles JNV, Wetherell K et al (2010) Coping style and depression influence the healing of diabetic foot ulcers: observational and mechanistic evidence. Diabetologia 53:1590-1598

2. Baron RM, Kenny DA (1986) The moderator-mediator variable distinction in social psychological research: conceptual, strategic, and statistical considerations. J Pers Soc Psychol 51:11731182

3. Bender R, Lange S (2001) Adjusting for multiple testing - when and how? J Clin Epidemiol 54:343-349

4. Bagley SC, White H, Golomb BA (2001) Logistic regression in the medical literature: standards for use and reporting, with particular attention to one medical domain. J Clin Epidemiol 54:979-985

5. Vittinghoff E, McCulloch CE (2006) Relaxing the rule of ten events per variable in logistic and Cox regression. Am J Epidemiol 156:710-718

6. Young MJ, Boulton AJM, Mcleod AF, Williams DRR, Sonksen PH (1993) A multicentre study of the prevalence of diabetic peripheral neuropathy in the UK hospital clinic population. Diabetologia $36: 150-154$

7. Piaggesi A, Viacava P, Rizzo L et al (2003) Semi-quantitative histological analysis of the histopathological features of the neuropathic foot ulcer - effects of pressure relief. Diabetes Care $26: 3123-3128$ 